\title{
MAIA TYPE FIXED POINT RESULTS FOR MULTIVALUED $F$-CONTRACTIONS
}

\author{
MURAT OLGUN, TUĞÇE ALYILDIZ, ÖZGE BIÇER, AND ISHAK ALTUN
}

Received 28 February, 2018

\begin{abstract}
In this paper, by considering the Wardowski's technique, we present fixed point results for multivalued mapping on a space with two metrics. Also, taking into account $\alpha$-admissibility of a multivalued mapping, we provide some more general results.
\end{abstract}

2010 Mathematics Subject Classification: 54H25; 47H10

Keywords: fixed point, multivalued $F$-contractions, complete metric space

\section{INTRODUCTION AND PRELIMINARIES}

In 2012, Wardowski [18] introduced a new concept for contraction mappings called $F$-contraction by considering a class of real valued functions. Let $\mathcal{F}$ be the set of all functions $F:(0, \infty) \longrightarrow \mathbb{R}$ satisfying the following conditions:

(F1) $F$ is strictly increasing, i.e., for all $\alpha, \beta \in(0, \infty)$ such that $\alpha<\beta$, $F(\alpha)<F(\beta)$,

(F2) For each sequence $\left\{a_{n}\right\}$ of positive numbers

$$
\lim _{n \rightarrow \infty} a_{n}=0 \Leftrightarrow \lim _{n \rightarrow \infty} F\left(a_{n}\right)=-\infty,
$$

(F3) There exists $k \in(0,1)$ such that $\lim _{\alpha \rightarrow 0^{+}} \alpha^{k} F(\alpha)=0$.

Then a self mapping $T$ of a metric space $(X, d)$ is said to be $F$-contraction if there exist $F \in \mathcal{F}$ and $\tau>0$ such that

$$
\forall x, y \in X, d(T x, T y)>0 \Rightarrow \tau+F(d(T x, T y)) \leq F(d(x, y)) .
$$

Taking different functions $F \in \mathcal{F}$ in (1.1) one gets a variety of $F$-contractions, some of them are of a type known in the literature. For example, let $F_{1}:(0, \infty) \rightarrow \mathbb{R}$ be given by the formula $F_{1}(\alpha)=\ln \alpha$. It is clear that $F_{1} \in \mathcal{F}$. Then each mapping $T: X \rightarrow X$ is an $F$-contraction such that

$$
d(T x, T y) \leq e^{-\tau} d(x, y) \text {, for all } x, y \in X \text { with } T x \neq T y .
$$

Therefore every Banach contraction mapping with contractive constant $0<L<1$ is an $F$-contraction with $F_{1}(\alpha)=\ln \alpha$ and $\tau=-\ln L>0$. Also by condition (F1), 
every $F$-contraction is a contractive mapping and hence it is continuous. From the Banach and Edelstein fixed point theorems, we know that every Banach contraction mapping on a complete metric space has a unique fixed point and every contractive mapping on a compact metric space has a unique fixed point. That is, passing from Banach to Edelstein fixed point theorem, when the class of mapping is expending by contractive condition, the structure of the space is restricted. Now, it may come to mind, is there any change of structure of the space when investigating the existence of the fixed points of $F$-contractions. Therefore, Wardowski [18] proved the following result without restricting the structure of the space:

Theorem 1. Let $(X, d)$ be a complete metric space and let $T: X \rightarrow X$ be an $F$ contraction. Then $T$ has a unique fixed point in $X$.

In the literature, there are many generalization of Theorem 1 (see $[4,7,11,12,17])$, one of them as follows:

Theorem 2 ([11]). Let $(X, d)$ be a complete metric space and let $T: X \rightarrow X$ be a mapping. If there exist $F \in \mathcal{F}$ and $\tau>0$ such that

$$
\forall x, y \in X, d(T x, T y)>0 \Rightarrow \tau+F(d(T x, T y)) \leq F(m(x, y)),
$$

where

$$
m(x, y)=\max \left\{d(x, y), d(x, T x), d(y, T y), \frac{1}{2}[d(x, T y)+d(y, T x)]\right\},
$$

then $T$ has a unique fixed point in $X$ provided that $T$ or $F$ is continuous.

It is our main aim in this work to give a fixed point theory for generalized multivalued $F$-contraction on a space with two metrics. First we recall some useful properties of multivalued mappings.

In 1969, using the concept of the Hausdorff metric, Nadler [13] introduced the notion of multivalued contraction mapping and proved a multivalued version of the well known Banach contraction principle. Let $(X, d)$ be a metric space. Denote by

- $P_{d}(X)$ the family of all nonempty subsets of $X$,

- $C_{d}(X)$ the family of all nonempty, closed subsets of $X$,

- $C B_{d}(X)$ the family of all nonempty, closed and bounded subsets of $X$,

- $K_{d}(X)$ the family of all nonempty, compact subsets of $X$.

It is well known that the function $H_{d}: C B_{d}(X) \times C B_{d}(X) \rightarrow \mathbb{R}$ defined by

$$
H_{d}(A, B)=\max \left\{\sup _{x \in A} d(x, B), \sup _{y \in B} d(y, A)\right\}
$$

for every $A, B \in C B_{d}(X)$, is a metric on $C B_{d}(X)$, which is called Hausdorff metric induced by $d$, where $d(x, B)=\inf \{d(x, y): y \in B\}$. Let $T: X \rightarrow C B_{d}(X)$ be a map, 
then $T$ is called a multivalued contraction if for all $x, y \in X$ there exists $L \in[0,1)$ such that

$$
H_{d}(T x, T y) \leq L d(x, y) .
$$

Nadler proved that every multivalued contraction mapping on a complete metric space has a fixed point [13].

Furthermore, let $X$ and $Y$ be two metric spaces. Then, a multivalued mapping $T: X \rightarrow P(Y)$ is said to be upper semicontinuous (lower semicontinuous) if the inverse image of closed sets (open sets) is closed (open). A multivalued mapping is continuous if it is upper as well as lower semicontinuous. $T$ is a closed multivalued mapping if the graph $G(T)=\{(x, y): x \in X, y \in T x\}$ is a closed subset of $X \times Y$. If $T$ is a closed multivalued mapping, then it is closed values. Conversely, if $T$ is both upper semicontinuous and closed values, then it is a closed multivalued mapping (see Proposition 2.17 of [9]).

Taking into account the ideas of Wardowski and Nadler, Altun et al [4] introduced the concept of multivalued F-contractions and obtained a fixed point result for these type mappings on complete metric space. After Acar et al in [1] presented the following definition and proved the following theorem:

Let $(X, d)$ be a metric space and $T: X \rightarrow C B_{d}(X)$ be a mapping. Then $T$ is said to be a generalized multivalued $F$-contraction with respect to $d$ if $F \in \mathcal{F}$ and there exists $\tau>0$ such that

$$
x, y \in X, H_{d}(T x, T y)>0 \Rightarrow \tau+F\left(H_{d}(T x, T y)\right) \leq F(M(x, y)),
$$

where

$$
M(x, y)=\max \left\{d(x, y), d(x, T x), d(y, T y), \frac{1}{2}[d(x, T y)+d(y, T x)]\right\} .
$$

Theorem 3 ([1]). Let $(X, d)$ be complete metric space and $T: X \rightarrow K_{d}(X)$ be a generalized multivalued $F$-contraction with respect to $d$. If $T$ or $F$ is continuous, then $T$ has a fixed point in $X$.

In 2012, Samet et al [16] introduced the concept of $\alpha$ - $\psi$-contractive and $\alpha$-admissible mapping and established various fixed point theorems. Also, Asl et al [5] defined the notion of $\alpha$-admissible and $\alpha_{*}$-admissible multivalued mappings as follows:

Let $(X, d)$ be a metric space, $T: X \rightarrow P_{d}(X)$ and $\alpha: X \times X \rightarrow[0, \infty)$ be a function. Then $T$ is said to be an $\alpha$-admissible mapping if for each $x \in X$ and $y \in T x$ with $\alpha(x, y) \geq 1$ implies $\alpha(y, z) \geq 1$ for all $z \in T y$ and $T$ is an $\alpha_{*}$-admissible mapping if for each $x \in X$ and $y \in T x$ with $\alpha(x, y) \geq 1$ implies $\alpha_{*}(T x, T y) \geq 1$, where $\alpha_{*}(T x, T y)=$ $\inf \{\alpha(a, b): a \in T x, b \in T y\}$. It is clear that an $\alpha_{*}$-admissible mapping is also $\alpha$ admissible.

Subsequently, in 2016, Durmaz and Altun [8] presented a fixed point results for $\alpha$-admissible multivalued mappings. 
Let $(X, d)$ be a metric space, $T: X \rightarrow C B_{d}(X)$ and $\alpha: X \times X \rightarrow[0, \infty)$ be two mappings. Define a set

$$
T_{\alpha}=\left\{(x, y): \alpha(x, y) \geq 1 \text { and } H_{d}(T x, T y)>0\right\} \subset X \times X .
$$

Given $F \in \mathcal{F}$, then $T$ is said to be a multivalued $(\alpha, F)$-contraction with respect to $d$ if there exists a function $\tau:(0, \infty) \rightarrow(0, \infty)$ such that

$$
\tau(d(x, y))+F\left(H_{d}(T x, T y)\right) \leq F(d(x, y))
$$

for all $(x, y) \in T_{\alpha}$. In this case, the function $\tau$ is called the contractive factor of $T$.

Theorem 4 ([8]). Let $(X, d)$ be a complete metric space and $T: X \rightarrow K_{d}(X)$ be an $\alpha$-admissible and multivalued $(\alpha, F)$-contraction with respect to $d$ with contractive factor $\tau$. Suppose that

$$
\liminf _{t \rightarrow s^{+}} \tau(t)>0, \text { for all } s \geq 0
$$

and there exist $x_{0} \in X$ and $x_{1} \in T x_{0}$ such that $\alpha\left(x_{0}, x_{1}\right) \geq 1$. If $T$ is a closed multivalued mapping, then $T$ has a fixed point.

On the other hand, fixed point theory studies can be established both for single valued and multivalued contraction type mappings on space with two metrics. Unlike the conventional, here it is accepted that the mapping is contraction or contraction type according to the one metric when the space is complete for the other metric. It can be find the fundamental version of these type fixed point results in $[2,6,10,14,15]$.

In this paper, we will present fixed point results for multivalued $F$-contraction mappings on a space with two metrics. Then, using $\alpha$-admissibility of a multivalued mapping, we will give some more general results.

\section{MAIN RESUltS}

Theorem 5. Let $(X, \rho)$ be a complete metric space, $d$ another metric on $X$ and $T: X \rightarrow K_{d}(X)$ be a generalized multivalued $F$-contraction with respect to d. Suppose that there exists $c>0$ such that

$$
\rho(x, y) \leq c d(x, y) \text { for each } x, y \in X .
$$

If $T$ is a closed multivalued mapping (with respect to $\rho$ ), then $T$ has a fixed point in $X$.

Proof. Let $x_{0} \in X$. Since $T x$ is nonempty for all $x \in X$, we can choose $x_{1} \in$ $T x_{0}$. If $x_{1} \in T x_{1}$, then $x_{1}$ is a fixed point of $T$ and so the proof is completed. Let $x_{1} \notin T x_{1}$. Then $d\left(x_{1}, T x_{1}\right)>0$ since $T x_{1}$ is closed. On the other hand, from (F1) and $d\left(x_{1}, T x_{1}\right) \leq H_{d}\left(T x_{0}, T x_{1}\right)$, we get

$$
F\left(d\left(x_{1}, T x_{1}\right)\right) \leq F\left(H_{d}\left(T x_{0}, T x_{1}\right)\right) .
$$

Since $T$ be a generalized multivalued $F$-contraction with respect to $d$, we obtain that

$$
F\left(d\left(x_{1}, T x_{1}\right)\right) \leq F\left(H_{d}\left(T x_{0}, T x_{1}\right)\right) \leq F\left(M\left(x_{0}, x_{1}\right)\right)-\tau
$$




$$
\begin{aligned}
& =F\left(\max \left\{\begin{array}{c}
d\left(x_{0}, x_{1}\right), d\left(x_{0}, T x_{0}\right), d\left(x_{1}, T x_{1}\right), \\
\frac{1}{2}\left[d\left(x_{0}, T x_{1}\right)+d\left(x_{1}, T x_{0}\right)\right]
\end{array}\right\}\right)-\tau \\
& \leq F\left(\max \left\{d\left(x_{0}, x_{1}\right), d\left(x_{1}, T x_{1}\right), \frac{1}{2} d\left(x_{0}, T x_{1}\right)\right\}\right)-\tau \\
& \leq F\left(\max \left\{\begin{array}{c}
d\left(x_{0}, x_{1}\right), d\left(x_{1}, T x_{1}\right), \\
\frac{1}{2}\left[d\left(x_{0}, x_{1}\right)+d\left(x_{1}, T x_{1}\right)\right]
\end{array}\right\}\right)-\tau \\
& =F\left(\max \left\{d\left(x_{0}, x_{1}\right), d\left(x_{1}, T x_{1}\right)\right\}\right)-\tau .
\end{aligned}
$$

If $d\left(x_{0}, x_{1}\right) \leq d\left(x_{1}, T x_{1}\right)$, then we have $F\left(d\left(x_{1}, T x_{1}\right)\right) \leq F\left(d\left(x_{1}, T x_{1}\right)\right)-\tau$, which is a contradiction since $\tau>0$. Thus we get

$$
F\left(d\left(x_{1}, T x_{1}\right)\right) \leq F\left(d\left(x_{0}, x_{1}\right)\right)-\tau .
$$

Since $T x_{1}$ is compact with respect to $d$, we obtain that $x_{2} \in T x_{1}$ such that $d\left(x_{1}, x_{2}\right)=$ $d\left(x_{1}, T x_{1}\right)$. Therefore, from (2.2)

$$
F\left(d\left(x_{1}, x_{2}\right)\right) \leq F\left(H_{d}\left(T x_{0}, T x_{1}\right)\right) \leq F\left(d\left(x_{0}, x_{1}\right)\right)-\tau .
$$

If we continue recursively, then we obtain a sequence $\left\{x_{n}\right\}$ in $X$ such that $x_{n+1} \in T x_{n}$ and

$$
F\left(d\left(x_{n}, x_{n+1}\right)\right) \leq F\left(d\left(x_{n}, x_{n-1}\right)-\tau\right.
$$

for all $n \in \mathbb{N}$. If there exists $n_{0} \in \mathbb{N}$ for which $x_{n_{0}} \in T x_{n_{0}}$, then $x_{n_{0}}$ is a fixed point of $T$. Therefore, suppose that for every $n \in \mathbb{N}, x_{n} \notin T x_{n}$. Denote $d_{n}=d\left(x_{n}, x_{n+1}\right)$, for $n=0,1,2, \ldots$. Then, $d_{n}>0$ for all $n$ and, using (2.3), the following holds:

$$
F\left(d_{n}\right) \leq F\left(d_{n-1}\right)-\tau \leq F\left(d_{n-2}\right)-2 \tau \leq \ldots \leq F\left(d_{0}\right)-n \tau .
$$

From (2.4), we obtain $\lim _{n \rightarrow \infty} F\left(d_{n}\right)=-\infty$. Hence, from (F2), we have

$$
\lim _{n \rightarrow \infty} d_{n}=0 .
$$

By (F3), there exists $k \in(0,1)$ such that

$$
\lim _{n \rightarrow \infty} d_{n}^{k} F\left(d_{n}\right)=0 \text {. }
$$

From (2.4), the following holds for all $n \in \mathbb{N}$

$$
d_{n}^{k} F\left(d_{n}\right)-d_{n}^{k} F\left(d_{0}\right) \leq-d_{n}^{k} n \tau \leq 0
$$

Letting $n \rightarrow \infty$ in (2.5), we obtain that

$$
\lim _{n \rightarrow \infty} n d_{n}^{k}=0 \text {. }
$$

Hence, there exists $n_{1} \in \mathbb{N}$ such that $n d_{n}^{k} \leq 1$ for all $n \geq n_{1}$. Therefore, we have

$$
d_{n} \leq \frac{1}{n^{1 / k}}
$$


for all $n \geq n_{1}$. In order to show that $\left\{x_{n}\right\}$ is a Cauchy sequence, consider $m, n \in \mathbb{N}$ such that $m>n \geq n_{1}$. By (2.6) and using the triangular inequality for the metric, we have

$$
\begin{aligned}
d\left(x_{n}, x_{m}\right) & \leq d\left(x_{n}, x_{n+1}\right)+d\left(x_{n+1}, x_{n+2}\right)+\ldots+d\left(x_{m-1}, x_{m}\right) \\
& =d_{n}+d_{n+1}+\ldots+d_{m-1} \\
& =\sum_{i=n}^{m-1} d_{i} \leq \sum_{i=n}^{\infty} d_{i} \leq \sum_{i=n}^{\infty} \frac{1}{i^{1 / k}} .
\end{aligned}
$$

From the convergence of the series $\sum_{i=1}^{\infty} \frac{1}{i^{1 / k}}$, we obtain $\lim _{n \rightarrow \infty} d\left(x_{n}, x_{m}\right)=0$. Thus $\left\{x_{n}\right\}$ is a Cauchy sequence in $(X, d)$. From (2.1) the sequence $\left\{x_{n}\right\}$ is a Cauchy in $(X, \rho)$ too. Since $(X, \rho)$ is a complete metric space, there exists $x \in X$ with $\rho\left(x_{n}, x\right) \rightarrow 0$ as $n \rightarrow \infty$.

Since $T$ is a closed multivalued mapping (with respect to $\rho$ ), we have $x \in T x$. Thus, $T$ has a fixed point in $X$.

Remark 1. If we take $d=\rho$ in Theorem 5, then Theorem 3 holds.

Corollary 1. Let $(X, \rho)$ be a complete metric space, $d$ another metric on $X$ which satisfies (2.1) and $T: X \rightarrow K_{d}(X)$ be a multivalued operator. Suppose that there exist $F \in \mathcal{F}$ and $\tau>0$ such that

$$
x, y \in X, H_{d}(T x, T y)>0 \Longrightarrow \tau+F\left(H_{d}(T x, T y)\right) \leq F(d(x, y)) .
$$

If $T$ is a closed multivalued mapping (with respect to $\rho$ ), then $T$ has a fixed point in $X$.

Remark 2. Note that in Theorem 5, Tx is compact for all $x \in X$. Thus, one can ask: Does $T$ has a fixed point if $T: X \rightarrow C B_{d}(X)$ is a generalized multivalued $F$ contraction? The following example, which is modified from Example 3.2 of [3], shows the answer is negative.

Example 1. Let $X=[0,1], \rho$ is the usual metric and

$$
d(x, y)=\left\{\begin{array}{ll}
0, & x=y \\
1+|x-y|, & x \neq y
\end{array} .\right.
$$

Then it is clear that $(X, \rho)$ is a complete metric space and $\rho(x, y) \leq d(x, y)$ for all $x, y \in X$. Since $\tau_{d}$ is discrete topology, then all subsets of $X$ are closed and also they are bounded. Therefore all subsets of $X$ are closed and bounded with respect to $d$. Define a map $T: X \rightarrow C B_{d}(X)$,

$$
T x=\left\{\begin{array}{cc}
Q, & x \in X \backslash Q \\
X \backslash Q, & x \in Q
\end{array},\right.
$$


where $Q$ is the set of all rational numbers in $X$. Therefore $T$ has no fixed point. Now, define $F:(0, \infty) \rightarrow \mathbb{R}$ by

$$
F(\alpha)=\left\{\begin{array}{cc}
\ln \alpha, & \alpha \leq 1 \\
\alpha, & \alpha>1
\end{array},\right.
$$

then we can see that $F \in \mathcal{F}$. Now we show that $T$ is a generalized multivalued $F$ contraction with respect to $d$, that is

$$
\forall x, y \in X\left[H_{d}(T x, T y)>0 \Rightarrow 1+F\left(H_{d}(T x, T y)\right) \leq F(M(x, y))\right] .
$$

Note that $H_{d}(T x, T y)>0 \Rightarrow\{x, y\} \cap Q$ is a singleton. Therefore for $x, y \in X$ with $H(T x, T y)>0$, we have

$$
H_{d}(T x, T y)=H_{d}(Q, X \backslash Q)=1<1+|x-y|=d(x, y) \leq M(x, y)
$$

and so

$$
1+F\left(H_{d}(T x, T y)\right) \leq F(M(x, y)) .
$$

However, by adding the following condition on $F$, we can consider $C B_{d}(X)$ instead of $K_{d}(X)$ in Theorem 5:

(F4) $F(\inf A)=\inf F(A)$ for all $A \subset(0, \infty)$ with $\inf A>0$.

Remark 3. Note that if $F$ is right-continuous and satisfies $(F 1)$, then it satisfies (F4).

Denote by $\mathcal{F}_{*}$, the set of all functions $F$ satisfying (F1)-(F4). It is clear that $\mathcal{F}_{*} \subset \mathcal{F}$.

Theorem 6. Let $(X, \rho)$ be a complete metric space, $d$ another metric on $X$ which satisfies (2.1) and $T: X \rightarrow C B_{d}(X)$ be a generalized multivalued $F$-contraction with $F \in \mathcal{F}_{*}$. If $T$ is a closed multivalued mapping (with respect to $\rho$ ), then $T$ has a fixed point in $X$.

Proof. Let $x_{0} \in X$. Since $T x$ is nonempty for all $x \in X$, we can choose $x_{1} \in T x_{0}$. If $x_{1} \in T x_{1}$, then $x_{1}$ is a fixed point of $T$ and so the proof is completed. Let $x_{1} \notin$ $T x_{1}$. Then $d\left(x_{1}, T x_{1}\right)>0$ since $T x_{1}$ is closed. On the other hand, from (F1) and $d\left(x_{1}, T x_{1}\right) \leq H_{d}\left(T x_{0}, T x_{1}\right)$, we get

$$
F\left(d\left(x_{1}, T x_{1}\right)\right) \leq F\left(H_{d}\left(T x_{0}, T x_{1}\right)\right) .
$$

Since $T$ be a generalized multivalued $F$-contraction, we can write that

$$
F\left(d\left(x_{1}, T x_{1}\right)\right) \leq F\left(H_{d}\left(T x_{0}, T x_{1}\right)\right) \leq F\left(M\left(x_{1}, x_{0}\right)\right)-\tau .
$$

By (F4), we get

$$
F\left(d\left(x_{1}, T x_{1}\right)\right)=\inf _{y \in T x_{1}} F\left(d\left(x_{1}, y\right)\right)
$$

since $d\left(x_{1}, T x_{1}\right)>0$. Hence, from (2.8) we have

$$
\inf _{y \in T x_{1}} F\left(d\left(x_{1}, y\right)\right) \leq F\left(M\left(x_{1}, x_{0}\right)\right)-\tau<F\left(M\left(x_{1}, x_{0}\right)\right)-\frac{\tau}{2} .
$$


Therefore, from (2.9) there exists $x_{2} \in T x_{1}$ such that

$$
F\left(d\left(x_{1}, x_{2}\right)\right) \leq F\left(M\left(x_{1}, x_{0}\right)\right)-\frac{\tau}{2} .
$$

Otherwise, by the same way we obtain $x_{3} \in T x_{2}$ such that

$$
F\left(d\left(x_{2}, x_{3}\right)\right) \leq F\left(M\left(x_{2}, x_{1}\right)\right)-\frac{\tau}{2} .
$$

We continue iterative, so we get a sequence $\left\{x_{n}\right\}$ in $X$ such that $x_{n+1} \in T x_{n}$ and for all $n=1,2,3, \ldots$

$$
F\left(d\left(x_{n}, x_{n+1}\right)\right) \leq F\left(M\left(x_{n}, x_{n-1}\right)\right)-\frac{\tau}{2} .
$$

The rest of the proof can be completed as in the proof of Theorem 5 .

Now, we present a new fixed point result for multivalued $F$-contraction by $\alpha$ admissibility of a multivalued mappings on space with two metrics.

Theorem 7. Let $(X, \rho)$ be a complete metric space, $d$ another metric on $X$ which satisfies (2.1) and $T: X \rightarrow K_{d}(X)$ be an $\alpha$-admissible and multivalued $(\alpha, F)$-contraction with respect to $d$ with contractive factor $\tau$ satisfying (1.5). Suppose that there exist $x_{0} \in X$ and $x_{1} \in T x_{0}$ such that $\alpha\left(x_{0}, x_{1}\right) \geq 1$. If $T$ is a closed multivalued mapping (with respect to $\rho)$, then $T$ has a fixed point.

Proof. Suppose that $T$ has no fixed point. Then $d(x, T x)>0$, for all $x \in X$. Let $x_{0}$ and $x_{1}$ be as mentioned in the hypothesis, then $H_{d}\left(T x_{0}, T x_{1}\right)>0$. So $\left(x_{0}, x_{1}\right) \in T_{\alpha}$, thus we can use (1.4) for $x_{0}$ and $x_{1}$. By (F1) we have

$$
F\left(d\left(x_{1}, T x_{1}\right)\right) \leq F\left(H_{d}\left(T x_{0}, T x_{1}\right)\right) \leq F\left(d\left(x_{1}, x_{0}\right)\right)-\tau\left(d\left(x_{1}, x_{0}\right)\right) .
$$

Since $T x_{1}$ is compact, there exists $x_{2} \in T x_{1}$ such that $d\left(x_{1}, x_{2}\right)=d\left(x_{1}, T x_{1}\right)$. Therefore, from (2.10)

$$
F\left(d\left(x_{1}, x_{2}\right)\right) \leq F\left(H_{d}\left(T x_{0}, T x_{1}\right)\right) \leq F\left(d\left(x_{1}, x_{0}\right)\right)-\tau\left(d\left(x_{1}, x_{0}\right)\right) .
$$

Now, since $T$ is an $\alpha$-admissible mapping we have $\alpha\left(x_{1}, x_{2}\right) \geq 1$ and also $H_{d}\left(T x_{1}, T x_{2}\right)>0$. Therefore, $\left(x_{1}, x_{2}\right) \in T_{\alpha}$, so we can use (1.4) for $x_{1}$ and $x_{2}$. Thus

$$
F\left(d\left(x_{2}, T x_{2}\right)\right) \leq F\left(H_{d}\left(T x_{1}, T x_{2}\right)\right) \leq F\left(d\left(x_{2}, x_{1}\right)\right)-\tau\left(d\left(x_{2}, x_{1}\right)\right) .
$$

Since $T x_{2}$ is compact, there exists $x_{3} \in T x_{2}$ such that $d\left(x_{2}, x_{3}\right)=d\left(x_{2}, T x_{2}\right)$. Therefore, we have

$$
F\left(d\left(x_{2}, x_{3}\right)\right) \leq F\left(H_{d}\left(T x_{1}, T x_{2}\right)\right) \leq F\left(d\left(x_{2}, x_{1}\right)\right)-\tau\left(d\left(x_{2}, x_{1}\right)\right) .
$$

By induction, we obtain a sequence $\left\{x_{n}\right\}$ in $X$ such that $x_{n+1} \in T x_{n},\left(x_{n}, x_{n+1}\right) \in T_{\alpha}$ and for all $n \in \mathbb{N}$

$$
F\left(d\left(x_{n}, x_{n+1}\right)\right) \leq F\left(d\left(x_{n}, x_{n-1}\right)\right)-\tau\left(d\left(x_{n}, x_{n-1}\right)\right) .
$$


Denote $d_{n}=d\left(x_{n}, x_{n+1}\right)$, for $n=0,1,2, \ldots$. Then, $d_{n}>0$ for all $n$ and, using (2.11), $\left\{d_{n}\right\}$ is decreasing and hence convergent. By (1.5), there exists $\delta>0$ and $n_{0} \in \mathbb{N}$ such that $\tau\left(d_{n}\right)>\delta$ for all $n>n_{0}$. Thus, we get

$$
\begin{aligned}
F\left(d_{n}\right) & \leq F\left(d_{n-1}\right)-\tau\left(d_{n-1}\right) \\
& \leq F\left(d_{n-2}\right)-\tau\left(d_{n-1}\right)-\tau\left(d_{n-2}\right) \\
& \vdots \\
& \leq F\left(d_{0}\right)-\tau\left(d_{n-1}\right)-\tau\left(d_{n-2}\right)-\cdots-\tau\left(d_{0}\right) \\
& \leq F\left(d_{0}\right)-\tau\left(d_{n-1}\right)-\tau\left(d_{n-2}\right)-\cdots-\tau\left(d_{n_{0}}\right) \\
& \leq F\left(d_{0}\right)-\left(n-n_{0}\right) \delta
\end{aligned}
$$

for all $n>n_{0}$. Letting $n \rightarrow \infty$ in the above inequality, we obtain $\lim _{n \rightarrow \infty} F\left(d_{n}\right)=-\infty$. Hence, from (F2), we have

$$
\lim _{n \rightarrow \infty} d_{n}=0 .
$$

By (F3), there exists $k \in(0,1)$ such that

$$
\lim _{n \rightarrow \infty} d_{n}^{k} F\left(d_{n}\right)=0 \text {. }
$$

From (2.12), the following holds for all $n>n_{0}$

$$
d_{n}^{k} F\left(d_{n}\right)-d_{n}^{k} F\left(d_{0}\right) \leq d_{n}^{k}\left[F\left(d_{0}\right)-\left(n-n_{0}\right) \delta\right]-d_{n}^{k} F\left(d_{0}\right)=-d_{n}^{k}\left(n-n_{0}\right) \boldsymbol{\delta} \leq 0 .
$$

Taking into account (2.13), we obtain that from the above inequality

$$
\lim _{n \rightarrow \infty} n d_{n}^{k}=0 \text {. }
$$

Hence, there exists $n_{1} \in \mathbb{N}$ such that $n d_{n}^{k} \leq 1$ for all $n \geq n_{1}$. Therefore, we have

$$
d_{n} \leq \frac{1}{n^{1 / k}} .
$$

for all $n \geq n_{1}$. In order to show that $\left\{x_{n}\right\}$ is a Cauchy sequence consider $m, n \in \mathbb{N}$ such that $m>n \geq n_{1}$. Then, we get

$$
\begin{aligned}
d\left(x_{n}, x_{m}\right) & \leq d\left(x_{n}, x_{n+1}\right)+d\left(x_{n+1}, x_{n+2}\right)+\ldots+d\left(x_{m-1}, x_{m}\right) \\
& <\sum_{i=n}^{\infty} d\left(x_{i}, x_{i+1}\right) \leq \sum_{i=n}^{\infty} \frac{1}{i^{1 / k}} .
\end{aligned}
$$

From the convergence of the series $\sum_{i=1}^{\infty} \frac{1}{i^{1 / k}}$, we obtain $\lim _{n \rightarrow \infty} d\left(x_{n}, x_{m}\right)=0$. Thus $\left\{x_{n}\right\}$ is a Cauchy sequence in $(X, d)$. From (2.1) the sequence $\left\{x_{n}\right\}$ is a Cauchy in $(X, \rho)$ too. Since $(X, \rho)$ is a complete metric space, there exists $x \in X$ with $\rho\left(x_{n}, x\right) \rightarrow 0$ as $n \rightarrow \infty$.

Since $T$ is closed multivalued mapping (with respect to $\rho$ ), then we have $x \in T x$, which is a contradiction. 
Therefore, $T$ has a fixed point in $X$.

Remark 4. We can take $C B_{d}(X)$ instead of $K_{d}(X)$ in Theorem 7 with $F \in \mathcal{F}_{*}$.

Theorem 8. Let $(X, \rho)$ be a complete metric space, $d$ another metric on $X$ which satisfies (2.1) and $T: X \rightarrow C B_{d}(X)$ be an $\alpha$-admissible and multivalued $(\alpha, F)$ contraction with $F \in \mathcal{F}_{*}$ and contractive factor $\tau$ satisfying (1.5). Suppose that there exist $x_{0} \in X$ and $x_{1} \in T x_{0}$ such that $\alpha\left(x_{0}, x_{1}\right) \geq 1$. If $T$ is a closed multivalued mapping (with respect to $\rho$ ), then $T$ has a fixed point.

Proof. We start as in the proof of Theorem 7. Considering the condition (F4), we get

$$
F\left(d\left(x_{1}, T x_{1}\right)\right)=\inf _{y \in T x_{1}} F\left(d\left(x_{1}, y\right)\right) .
$$

Therefore

$$
F\left(d\left(x_{1}, T x_{1}\right)\right) \leq F\left(H_{d}\left(T x_{0}, T x_{1}\right)\right) \leq F\left(d\left(x_{1}, x_{0}\right)\right)-\tau\left(d\left(x_{1}, x_{0}\right)\right),
$$

and we can write

$$
\inf _{y \in T x_{1}} F\left(d\left(x_{1}, y\right)\right) \leq F\left(d\left(x_{1}, x_{0}\right)\right)-\tau\left(d\left(x_{1}, x_{0}\right)\right)<F\left(d\left(x_{1}, x_{0}\right)\right)-\frac{\tau\left(d\left(x_{1}, x_{0}\right)\right)}{2} .
$$

Thus, there exists $x_{2} \in T x_{1}$ such that

$$
F\left(d\left(x_{1}, x_{2}\right)\right) \leq F\left(d\left(x_{1}, x_{0}\right)\right)-\frac{\tau\left(d\left(x_{1}, x_{0}\right)\right)}{2} .
$$

The rest of the proof can be completed as in the proof of Theorem 7.

\section{ACKNOWLEDGEMENT}

The authors are thankful to the referees for making valuable suggestions leading to a better presentation of the paper.

\section{REFERENCES}

[1] Ö. Acar, G. Durmaz, and G. Minak, "Generalized multivalued $F$-contractions on complete metric spaces.” Bull. Iranian Math. Soc., vol. 40, no. 6, pp. 1469-1478, 2014.

[2] R. P. Agarwal and D. O'Regan, "Fixed point theory for generalized contractions on space with two metrics." J. Math. Anal. Appl., vol. 248, no. 2, pp. 402-414, 2000, doi: 10.1006/jmaa.2000.6914.

[3] I. Altun, G. Durmaz, G. Minak, and S. Romaguera, "Multivalued almost $F$-contractions on complete metric spaces." Filomat, vol. 30, no. 2, pp. 441-448, 2016, doi: 10.2298/FIL1602441A.

[4] I. Altun, G. Mınak, and H. Dağ, "Multivalued $F$-contractions on complete metric space." J. Nonlinear Convex Anal., vol. 16, no. 4, pp. 659-666, 2015.

[5] J. H. Asl, S. Rezapour, and N. Shahzad, "On fixed points of $\alpha-\psi$-contractive multifunctions." Fixed Point Theory Appl., vol. 2012, no. 6, 2012, doi: 10.1186/1687-1812-2012-212.

[6] V. Berinde, Iterative approximation of fixed points. Springer-Verlag, Berlin Heidelberg, 2007. doi: 10.1007/978-3-540-72234-2.

[7] M. Cosentino and P. Vetro, "Fixed point results for $F$-contractive mappings of Hardy-Rogerstype." Filomat, vol. 28, no. 4, pp. 715-722, 2014, doi: 10.2298/FIL1404715C. 
[8] G. Durmaz and I. Altun, "Fixed point results for $\alpha$-admissible multivalued $F$-contractions." Miskolc Math. Notes, vol. 17, no. 1, pp. 187-199, 2016, doi: 10.18514/MMN.2016.1478.

[9] S. Hu and N. S. Papageorgiou, Handbook of multivalued analysis. Kluwer Academic Publishers, 1997, vol. I.

[10] M. G. Maia, "Un'obsservazione sulle contrazioni metriche." Rend. Sem. Mat. Uni. Padova, vol. 40, no. 139-143, 1968.

[11] G. Mınak, A. Helvacı, and I. Altun, "Ćirić type generalized $F$-contractions on complete metric spaces and fixed point results." Filomat, vol. 28, no. 6, pp. 1143-1151, 2014, doi: 10.2298/FIL1406143M.

[12] G. Minak, M. Olgun, and I. Altun, "A new approach to fixed point theorems for multivalued contractive maps." Carpathian J. Math., vol. 31, no. 2, pp. 241-248, 2015.

[13] S. B. Nadler, "Multi-valued contraction mappings." Pacific J. Math., vol. 30, pp. 475-488, 1969.

[14] D. O'Regan, A. Petrusel, and T. P. Petru, "Fixed point results for Ciric type contractions on a set with two separating gauge structures." Sci. Math. Jpn., vol. 68, no. 3, pp. 361-369, 2008.

[15] A. Petrusel and I. A. Rus, "Fixed point theory for multivalued operators on a set with two metrics." Fixed Point Theory, vol. 8, no. 1, pp. 97-104, 2007.

[16] B. Samet, C. Vetro, and P. Vetro, "Fixed point theorems for $\alpha-\psi$-contractive type mappings." Nonlinear Anal., vol. 75, pp. 2154-2165, 2012, doi: 10.1016/j.na.2011.10.014.

[17] M. Sgroi and C. Vetro, "Multi-valued $F$-contractions and the solution of certain functional and integral equations." Filomat, vol. 27, no. 7, pp. 1259-1268, 2013, doi: 10.2298/FIL1307259S.

[18] D. Wardowski, "Fixed points of a new type of contractive mappings in complete metric spaces." Fixed Point Theory Appl., vol. 2012, no. 94, 2012, doi: 10.1186/1687-1812-2012-94.

Authors' addresses

Murat Olgun

Department of Mathematics, Faculty of Science, Ankara University, 06100, Tandogan, Ankara, Turkey

E-mail address: olgun@ankara.edu.tr

Tuğçe Alyıldız

Department of Mathematics, Faculty of Science, Ankara University, 06100, Tandogan, Ankara, Turkey

E-mail address: tugcekavuzlu@hotmail.com

Özge Biçer

Department of Electronic Communication Technology, Vocational School, Istanbul Medipol University, Istanbul, Turkey

E-mail address: ozgeb89@hotmail.com

Ishak Altun

(Corresponding author) Department of Mathematics, Faculty of Science and Arts, Kirikkale University, 71450 Yahsihan, Kirikkale, Turkey

E-mail address: ishakaltun@yahoo.com 